\title{
Some first-order and higher-order statistical properties of polarization speckle
}

\author{
Xiao, Shengzhu; Nie, Jianlin; Hanson, Steen G.; Takeda, Mitsuo; Wang, Wei
}

Published in:

Proceedings of SPIE

Link to article, DOI:

$10.1117 / 12.2600101$

Publication date:

2021

Document Version

Publisher's PDF, also known as Version of record

Link back to DTU Orbit

Citation (APA):

Xiao, S., Nie, J., Hanson, S. G., Takeda, M., \& Wang, W. (2021). Some first-order and higher-order statistical properties of polarization speckle. In Proceedings of SPIE (Vol. 11875). [118750R] SPIE - International Society for Optical Engineering. Proceedings of SPIE - The International Society for Optical Engineering https://doi.org/10.1117/12.2600101

\section{General rights}

Copyright and moral rights for the publications made accessible in the public portal are retained by the authors and/or other copyright owners and it is a condition of accessing publications that users recognise and abide by the legal requirements associated with these rights.

- Users may download and print one copy of any publication from the public portal for the purpose of private study or research.

- You may not further distribute the material or use it for any profit-making activity or commercial gain

- You may freely distribute the URL identifying the publication in the public portal 


\section{Some first-order and higher-order statistical properties of polarization speckle}

Xiao, Shengzhu, Nie, Jianlin, Hanson, Steen G., Takeda, Mitsuo, Wang, Wei

Shengzhu Xiao, Jianlin Nie, Steen G. Hanson, Mitsuo Takeda, Wei Wang, "Some first-order and higher-order statistical properties of polarization speckle," Proc. SPIE 11875, Computational Optics 2021, 118750R (13 September 2021); doi: 10.1117/12.2600101

SPIE. Event: SPIE Optical Systems Design, 2021, Online Only 


\title{
Some first-order and higher-order statistical properties of polarization speckle
}

\author{
Shengzhu Xiao, ${ }^{a}$ Jianlin Nie, ${ }^{a}$ Steen G. Hanson, ${ }^{b}$ Mitsuo Takeda, ${ }^{\text {a,c }}$ and Wei Wang a,d \\ a International Center for Optical Research and Education (iCORE), Xi'an Technological University, Xi'an, \\ Shaanxi, China \\ b DTU Fotonik, Department of Photonics Engineering, Technical University of Denmark, Dk-4000 Roskilde, \\ Denmark \\ ${ }^{\mathrm{c}}$ Center for Optical Research and Education (CORE), Utsunomiya University, Utsunomiya, Tochigi, Japan \\ ${ }^{\mathrm{d}}$ School of Engineering and Physical Sciences, Heriot-Watt University, Edinburgh, EH14 4AS, UK
}

\begin{abstract}
In this paper, we revisit the role of polarization and coherence in the study of polarization speckle. It is shown that the type of polarization and coherence used in such analysis has an important difference from the "classical" polarization and coherence concepts. The analogy between polarization speckle and partially polarized thermal radiation is explored. We propose a concept referred to as ensemble-average polarization and coherence for statistical optics, and give the definition and physical indication for polarization speckle. Some statistical properties associated with polarization speckle including the 1st order statistics of the Stokes parameters and the ensemble-average Van Cittert-Zernike theorem for the ensembled-averaged Generalized Stokes parameters are investigated theoretically and experimentally to demonstrate their link and different physical features as compared with the conventional concepts.
\end{abstract}

Keywords: Polarization speckle, ensemble-average polarization and coherence, Generalized Stokes parameters

\section{INTRODUCTION}

In statistical physics, especially statistical optics, it's common to model light from a source other than a laser as a stationary and ergodic random process in time. ${ }^{[1,2]}$ Polarization and coherence measures are then defined by infinite time average, although statistical averages can be used when necessary and yield the same results as time averages. While, there exists an important class of problems for which the light is actually nonergodic over the ensemble of interest; that is, time and ensemble averages no longer yield the same results. For such problems, polarization and coherence concepts must be defined in terms of ensemble averages. On the other hand, since the emergence of the laser, people have explored the basic characteristics and extensive applications of continuous laser sources. Laser speckle is a kind of granular pattern with high contrast and small scale. Laser speckle is caused by scattering or scattering off a rough surface by a field from a linearly polarized laser ${ }^{[3,4]}$ Although the polarization changes after reflection or scattering have been studied for a long time, the statistical properties of scalar speckle and partial polarization speckle have been limited for a long time ${ }^{[4,10]}$. Recently, a speckle phenomenon associated with random polarization has been observed. We will introduce a new concept, polarized speckle, rather than the well-known partial polarization speckle. In this paper, the statistical properties of fully polarized speckle are studied, and the concepts of ensemble-average Generalized Stokes and ensemble-average Stokes are introduced. The statistical properties of first order polarization speckle are studied theoretically and experimentally. The statistical properties of the first-order polarization speckle are studied by using the narrow stokes parameter. The higher order statistical properties of polarized speckle are studied by using the Generalized Stokes parameter autocorrelation function.

\section{MANIFESTATIONS OF POLARIZATION SPECKLE}

In statistical physics, especially statistical optics, it's common to model light from a source other than a laser as a stationary and ergodic random process in time ${ }^{[1,2]}$. Polarization and coherence measures are then defined by infinite time averages, although statistical averages can be used when necessary and yield the same results as time averages. Nevertheless, there exists an important class of problems for which the light is actually nonergodic over the ensemble of 
interest; that is, time and ensemble averages no longer yield the same results. For such problems, polarization and coherence concepts must be defined in terms of ensemble averages.

Let the light from a highly coherent laser with a linear polarization illuminate a stationary random birefringence screen, and consider the polarization and coherence of light some distance beyond the screen. The intensity and polarization observed behind the random birefringence screen are extremely complex and its intensity and state-of-polarization result in a highly unpredictable pattern as shown in Fig.1(b) due to the complex and unknown microstructure of the random birefringence screen itself. If the random birefringence screen is moving, the electric field, intensity, and polarization behind the diffuser would fluctuate with time, and the usual definitions of polarization and coherence in terms of time average would be appropriate ${ }^{[4]}$. However, the random birefringence screen is not moving in the current situation of interest.

Because the electric field, intensity and state-of-polarization of the light behind the random birefringence screen are extremely complex and unpredictable, it is appropriate to model them as random process. However, by our usual definitions based on time averages ${ }^{[8]}$, it is not difficult to show that the light behind the random birefringence screen is completely polarized and completely coherent everywhere, giving that it is illuminated by completely polarized and completely coherent light. Such random electric field is referred to as the polarization speckle with its unique features of fluctuating state-of-polarization for a fully coherent light. A modified Young's interference experiment with an appropriate phase retarder placed behind one of the double pinholes will yield fringes with unity visibility indicating it's fully coherent everywhere. Similarly, the experimental measurement of the Stokes parameters giving the degree of polarization equal to one everywhere serves as evidence of fully polarized light.

Since a statistical treatment of such light seems appropriate and is indeed useful, the question then becomes over what statistical ensemble is the light a random process and how might we modify our definitions of polarization and coherence for such light. The ensemble to be considered is an ensemble of different random birefringence screens, each screen having a different microstructure but all screens having identical statistical properties over the ensemble. Statistical averages are then carried out over the ensemble of random birefringence screens, allowing the concepts of polarization and coherence to be defined.

For a single random birefringent screen, the observed light is completely polarized and fully coherent in the timeaverage sense, and the electric field in the observation plane can be represented by

$$
\mathbf{E}(\mathbf{r}, t)=\left[\tilde{E}_{x}(\mathbf{r}) \hat{x}+\tilde{E}_{y}(\mathbf{r}) \hat{y}\right] \exp (-j 2 \pi v t),
$$

where $v$ is the frequency of the monochromatic source. Note that the complex polarization vector $\tilde{E}_{x}(\mathbf{r}) \hat{x}+\tilde{E}_{y}(\mathbf{r}) \hat{y}$ is independent of time and in fact, represents the polarization phasor of the electric field. Using the conventional description for partially polarized light based on time-average definition of polarization, the $2 \times 2$ mutual coherence matrix of such light takes the form ${ }^{[6]}$

$$
\begin{aligned}
\mathbf{W}\left(\mathbf{r}_{1}, \mathbf{r}_{2}\right) & =\left[\begin{array}{ll}
<\tilde{E}_{x}^{*}\left(\mathbf{r}_{1}, t\right) \tilde{E}_{x}\left(\mathbf{r}_{2}, t\right)> & <\tilde{E}_{x}^{*}\left(\mathbf{r}_{1}, t\right) \tilde{E}_{y}\left(\mathbf{r}_{2}, t\right)> \\
<\tilde{E}_{y}^{*}\left(\mathbf{r}_{1}, t\right) \tilde{E}_{x}\left(\mathbf{r}_{2}, t\right)> & <\tilde{E}_{y}^{*}\left(\mathbf{r}_{1}, t\right) \tilde{E}_{y}\left(\mathbf{r}_{2}, t\right)>
\end{array}\right] \\
& =\left[\begin{array}{ll}
\tilde{E}_{x}^{*}\left(\mathbf{r}_{1}\right) \tilde{E}_{x}\left(\mathbf{r}_{2}\right) & \tilde{E}_{x}^{*}\left(\mathbf{r}_{1}\right) \tilde{E}_{y}\left(\mathbf{r}_{2}\right) \\
\tilde{E}_{y}^{*}\left(\mathbf{r}_{1}\right) \tilde{E}_{x}\left(\mathbf{r}_{2}\right) & \tilde{E}_{y}^{*}\left(\mathbf{r}_{1}\right) \tilde{E}_{y}\left(\mathbf{r}_{2}\right)
\end{array}\right] .
\end{aligned}
$$

Here angular brackets indicate "time average". This is the expected form of the mutual coherence matrix for a completed polarized and fully coherent light. Suppose, however, that rather than averaging with respect to time, instead, we average over an ensemble of statistically similar random birefringence screens, indicated by placing a bar over the symbol $\mathbf{W}$. The mutual coherence matrix so defined takes the form:

$$
\begin{aligned}
\overline{\mathbf{W}}\left(\mathbf{r}_{1}, \mathbf{r}_{2}\right) & =\left[\begin{array}{ll}
\mathcal{E}\left\{\tilde{E}_{x}^{*}\left(\mathbf{r}_{1}, t\right) \tilde{E}_{x}\left(\mathbf{r}_{2}, t\right)\right\} & \mathcal{E}\left\{\tilde{E}_{x}^{*}\left(\mathbf{r}_{1}, t\right) \tilde{E}_{y}\left(\mathbf{r}_{2}, t\right)\right\} \\
\mathcal{E}\left\{\tilde{E}_{y}^{*}\left(\mathbf{r}_{1}, t\right) \tilde{E}_{x}\left(\mathbf{r}_{2}, t\right)\right\} & \mathcal{E}\left\{\tilde{E}_{y}^{*}\left(\mathbf{r}_{1}, t\right) \tilde{E}_{y}\left(\mathbf{r}_{2}, t\right)\right\}
\end{array}\right] \\
& =\left[\begin{array}{ll}
\overline{\tilde{E}_{x}^{*}\left(\mathbf{r}_{1}\right) \tilde{E}_{x}\left(\mathbf{r}_{2}\right)} & \overline{\tilde{E}_{x}^{*}\left(\mathbf{r}_{1}\right) \tilde{E}_{y}\left(\mathbf{r}_{2}\right)} \\
\overline{\tilde{E}_{y}^{*}\left(\mathbf{r}_{1}\right) \tilde{E}_{x}\left(\mathbf{r}_{2}\right)} & \overline{\tilde{E}_{y}^{*}\left(\mathbf{r}_{1}\right) \tilde{E}_{y}\left(\mathbf{r}_{2}\right)}
\end{array}\right],
\end{aligned}
$$


where both $\mathcal{E}\{\cdots\}$ and over bar represent a statistical expectation. In general, $\overline{\mathbf{W}}\left(\mathbf{r}_{1}, \mathbf{r}_{2}\right) \neq \mathbf{W}\left(\mathbf{r}_{1}, \mathbf{r}_{2}\right)$ in the situation of our interest here. Note the fact that polarization can be described by a $2 \times 2$ equal location and equal time correlation matrix: coherency matrix. Therefore, the new matrix referred to as the ensemble-average coherency matrix can be defined as

$$
\begin{aligned}
& \overline{\mathbf{J}(\mathbf{r})}=\overline{\mathbf{W}}\left(\mathbf{r}_{1}=\mathbf{r}_{2}=\mathbf{r}\right)=\left[\begin{array}{ll}
\overline{\tilde{E}_{x}^{*}(\mathbf{r}) \tilde{E}_{x}(\mathbf{r})} & \overline{\tilde{E}_{x}^{*}(\mathbf{r}) \tilde{E}_{y}(\mathbf{r})} \\
\overline{\tilde{E}_{y}^{*}(\mathbf{r}) \tilde{E}_{x}(\mathbf{r})} & \overline{\tilde{E}_{y}^{*}(\mathbf{r}) \tilde{E}_{y}(\mathbf{r})}
\end{array}\right]
\end{aligned}
$$

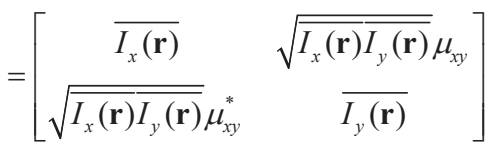

Similar to the concept of ensemble-average coherence introduced by Goodman to study the laser speckle ${ }^{[6]}$, the proposed concepts of ensemble-average polarization and coherence will be found particularly useful for polarization speckle. We can also introduce the ensemble-average Stokes parameters in a similar way. That is

$$
\begin{aligned}
& \overline{S_{0}(\mathbf{r})}=\left[\overline{\tilde{E}_{x}^{*}(\mathbf{r}) \tilde{E}_{x}(\mathbf{r})}+\overline{\tilde{E}_{y}^{*}(\mathbf{r}) \tilde{E}_{y}(\mathbf{r})}\right] / 2, \\
& \overline{S_{1}(\mathbf{r})}=\left[\overline{\tilde{E}_{x}^{*}(\mathbf{r}) \tilde{E}_{x}(\mathbf{r})}-\overline{\left.\tilde{E}_{y}^{*}(\mathbf{r}) \tilde{E}_{y}(\mathbf{r})\right]} / 2,\right. \\
& \overline{S_{2}(\mathbf{r})}=\left[\overline{\tilde{E}_{x}^{*}(\mathbf{r}) \tilde{E}_{y}(\mathbf{r})}+\overline{\tilde{E}_{y}^{*}(\mathbf{r}) \tilde{E}_{x}(\mathbf{r})}\right] / 2, \\
& \overline{S_{3}(\mathbf{r})}=\left[\overline{\tilde{E}_{x}^{*}(\mathbf{r}) \tilde{E}_{y}(\mathbf{r})}-\overline{\tilde{E}_{y}^{*}(\mathbf{r}) \tilde{E}_{x}(\mathbf{r})}\right] /(2 j) .
\end{aligned}
$$

Following the convention for the definition of the degree of polarization, we can introduce the ensemble-average degree of polarization as the ratio of the intensity in the uniformly polarized wave component to the total intensity of the wave,

$$
\overline{\mathcal{P}}=\sqrt{1-4 \operatorname{det}(\overline{\mathbf{J}}) /[\operatorname{tr}(\overline{\mathbf{J}})]^{2}} .
$$

Note that the ensemble-average degree of polarization always lies between 1 for a wave with uniform state-of polarization and 0 for a wave with completely random distribution for the state-of-polarization. Similarly, the ensembleaverage Generalized Stokes parameter can be defined as

$$
\begin{aligned}
& \overline{S_{0}\left(\mathbf{r}_{1}, \mathbf{r}_{2}\right)}=\left[\overline{\tilde{E}_{x}^{*}\left(\mathbf{r}_{1}\right) \tilde{E}_{x}\left(\mathbf{r}_{2}\right)}+\overline{\tilde{E}_{y}^{*}\left(\mathbf{r}_{1}\right) \tilde{E}_{y}\left(\mathbf{r}_{2}\right)}\right] / 2, \\
& \overline{S_{1}\left(\mathbf{r}_{1}, \mathbf{r}_{2}\right)}=\left[\overline{\tilde{E}_{x}^{*}\left(\mathbf{r}_{1}\right) \tilde{E}_{x}\left(\mathbf{r}_{2}\right)}-\overline{\tilde{E}_{y}^{*}\left(\mathbf{r}_{1}\right) \tilde{E}_{y}\left(\mathbf{r}_{2}\right)}\right] / 2, \\
& \overline{S_{2}\left(\mathbf{r}_{1}, \mathbf{r}_{2}\right)}=\left[\overline{\tilde{E}_{x}^{*}\left(\mathbf{r}_{1}\right) \tilde{E}_{y}\left(\mathbf{r}_{2}\right)}+\overline{\tilde{E}_{y}^{*}\left(\mathbf{r}_{1}\right) \tilde{E}_{x}\left(\mathbf{r}_{2}\right)}\right] / 2, \\
& \overline{S_{3}\left(\mathbf{r}_{1}, \mathbf{r}_{2}\right)}=\left[\overline{\tilde{E}_{x}^{*}\left(\mathbf{r}_{1}\right) \tilde{E}_{y}\left(\mathbf{r}_{2}\right)}-\overline{\tilde{E}_{y}^{*}\left(\mathbf{r}_{1}\right) \tilde{E}_{x}\left(\mathbf{r}_{2}\right)}\right] /(2 j) .
\end{aligned}
$$

\section{EXPERIMENTAL INVESTIGATION OF STATISTICS OF POLARIZATION SPECKLE}

\subsection{Detection of polarization speckle with the Oka-Ohtsuka polarization interferometer}

To generate and detect polarized speckles, a polar-interferometer referred to as the Oka-Ohtsuka interferometer is used, as shown in the figure $1 .{ }^{[12]}$ Linearly polarized light from a He-Ne laser source is introduced into a Mach-Zehnder interferometer, which is then divided into two components by the beam splitter BS1. The linearly polarized light reflected from BS1 is collimated through the microscope objective (MO2) and lens L2 to serve as the reference beam for the interference. The collimating reference beam is divided into two orthogonal and linearly polarized components in a polarized beam splitter (PBS). To balance the intensity of these two orthogonal components, a half-wave plate (HWP) was inserted in front of PBS to change the plane of polarization of the incident beam. The reflection component passes through the quarter-wave plate QWP1 to the tilted mirror (M2). When the beam is reflected, the tilt angle gives the spatial carrier frequency of the reflected beam. Similarly, another beam reflected on mirror M3, with the help of another 
quarter wave plate QWP2, is fully reflected on PBS. Thus, the reference beam from PBS to beam splitter BS2 consists of two orthogonal linear polarization components with different spatial carrier frequencies. On the other hand, a depolarizer DP is inserted into the other arm of the interferometer to depolarize the incident beam to serve as a signal wave and to control the degree of spatial polarization dependent on its rotation. To adjust the size of the polarized speckle, we slide a 10x objective lens (MO1) back and forth on a frosted glass plate (GG) to produce a suitable spot size. A reference beam with different spatial carrier frequencies with two orthogonal linear polarization components is interfered by the polarized speckle generated by the collimation of L2 lens. A CCD camera records the interferogram of polarizing speckles in the far field ${ }^{[13]}$.

By applying the Fourier fringe analysis technique to two different spatial carrier frequencies ${ }^{[14]}$, we recover the electric field components $\tilde{E}_{x}(\mathbf{r})$ and $\tilde{E}_{y}(\mathbf{r})$ from the two groups of fringe interferograms recorded, and obtain the required Stokes distribution for polarization speckle based on their definitions.

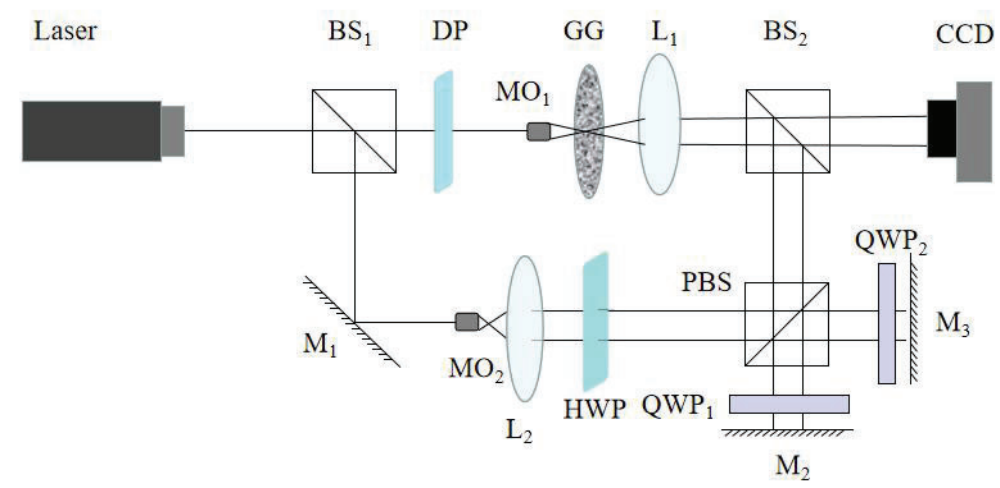

Figure 1. Experimental setup for detection of polarization speckle

\subsection{First-Order statistics of the Stokes parameters for polarization speckle}

Now, let us consider the first-order statistics of polarized speckle. Here, "first order" represents the statistical properties of a single point in space. Polarization speckle is generated by superposition of several random polarization phasors' contribution with fluctuating state-of-polarization in space. The probability density functions of the four Stokes parameters for polarization speckle have been derived. ${ }^{[4,7]}$ They are

$$
\begin{gathered}
p\left(S_{0}\right)=\frac{1}{\overline{\mathcal{P}} \overline{S_{0}}}\left[\exp \left(-\frac{2}{1+\overline{\mathcal{P}}} \frac{S_{0}}{\overline{S_{0}}}\right)-\exp \left(-\frac{2}{1-\overline{\mathcal{P}}} \frac{S_{0}}{\overline{S_{0}}}\right)\right], \\
p\left(S_{1}\right)=\frac{1}{\sqrt{\left(\overline{I_{x}}+\overline{I_{y}}\right)^{2}-4 \overline{I_{x}} \overline{I_{y}}\left|\mu_{x y}\right|^{2}}} \exp \left[\frac{-\left|S_{1}\right| \sqrt{\left(\overline{I_{x}}+\overline{I_{y}}\right)^{2}-4 \overline{I_{x}} \overline{I_{y}}\left|\mu_{x y}\right|^{2}}+S_{1}\left(\overline{I_{x}}-\overline{I_{y}}\right)}{\left.2 \overline{I_{x} \overline{I_{y}}\left(1-\left|\mu_{x y}\right|^{2}\right)}\right],}\right. \\
p\left(S_{2}\right)=\frac{1}{\sqrt{\left(\overline{I_{45}}+\overline{I_{135}}\right)^{2}-4 \overline{I_{45}} \overline{I_{135}}\left|\mu_{45,135}\right|^{2}}} \exp \left[\frac{-\left|S_{2}\right| \sqrt{\left(\overline{I_{45}}+\overline{I_{135}}\right)^{2}-4 \overline{I_{45}} \overline{I_{135}}\left|\mu_{45,135}\right|^{2}}+S_{2}\left(\overline{I_{45}}-\overline{I_{135}}\right)}{2 \overline{I_{45}} \overline{I_{135}}\left(1-\left|\mu_{45,135}\right|^{2}\right)}\right],
\end{gathered}
$$




$$
p\left(S_{3}\right)=\frac{1}{\sqrt{\left(\overline{I_{l c}}+\overline{I_{r c}}\right)^{2}-4 \overline{I_{l c}} \overline{I_{r c}}\left|\mu_{l c, c}\right|^{2}}} \exp \left[\frac{-\left|S_{3}\right| \sqrt{\left(\overline{\bar{I}_{l c}}+\overline{I_{r c}}\right)^{2}-4 \overline{I_{l c} I_{r c}}\left|\mu_{l c, r c}\right|^{2}}+S_{3}\left(\overline{I_{l c}}-\overline{I_{r c}}\right)}{2 \overline{I_{l c}} \overline{I_{r c}}\left(1-\left|\mu_{l c, r c}\right|^{2}\right)}\right],
$$

where

$$
\begin{aligned}
\overline{I_{45}}=\left[\overline{I_{x}}+\overline{I_{y}}+2 \sqrt{\bar{I}_{x} \overline{I_{y}}} \operatorname{Re}\left(\mu_{x y}\right)\right] / 2 ; & \overline{I_{135}}=\left[\overline{I_{x}}+\overline{I_{y}}-2 \sqrt{\overline{I_{x} \bar{I}_{y}}} \operatorname{Re}\left(\mu_{x y}\right)\right] / 2 ; \\
\sqrt{\overline{I_{45} \overline{I_{135}}}} \mu_{45,135}=\left[-\overline{I_{x}}+\overline{I_{y}}+2 j \sqrt{\overline{I_{x} \overline{I_{y}}}} \operatorname{Im}\left(\mu_{x y}\right)\right] / 2 ; & \sqrt{\bar{I}_{45} \overline{I_{135}}} \mu_{45,135}^{*}=\left[-\overline{I_{x}}+\overline{I_{y}}-2 j \sqrt{\overline{I_{x} \bar{I}_{y}}} \operatorname{Im}\left(\mu_{x y}\right)\right] / 2 ; \\
\overline{I_{l c}}=\left[\overline{I_{x}}+\overline{I_{y}}+2 \sqrt{\overline{I_{x}} \overline{\overline{I_{y}}}} \operatorname{Im}\left(\mu_{x y}\right)\right] / 2 ; & \overline{I_{r c}}=\left[\overline{I_{x}}+\overline{I_{y}}-2 \sqrt{\overline{I_{x}} \overline{I_{y}}} \operatorname{Im}\left(\mu_{x y}\right)\right] / 2 ; \\
\sqrt{\overline{I_{l c} \overline{I_{r c}}}} \mu_{l r}=\left[-\overline{I_{x}}+\overline{I_{y}}+2 j \sqrt{\overline{I_{x} \overline{I_{y}}}} \operatorname{Re}\left(\mu_{x y}\right)\right] / 2 ; & \sqrt{\overline{I_{45} \overline{I_{135}}}} \mu_{45,135}^{*}=\left[-\overline{I_{x}}+\overline{I_{y}}-2 j \sqrt{\overline{I_{x}} \overline{I_{y}}} \operatorname{Re}\left(\mu_{x y}\right)\right] / 2 .
\end{aligned}
$$

From the reconstructed electric fields for polarization speckle, we can calculate the Stokes parameters based on their definition and carry out the statistical analysis for these four reconstructed Stokes parameters. Figure 2(a) shows the distribution of $S_{0}$ as a function of the normalized value of $\overline{S_{0}}$. The histograms for the Stokes parameters $S_{1}, S_{2}$ and $S_{3}$ have been given in Fig. 2 (b)-(d), respectively, and they looks more like asymmetric Laplacian distributions from these examples.
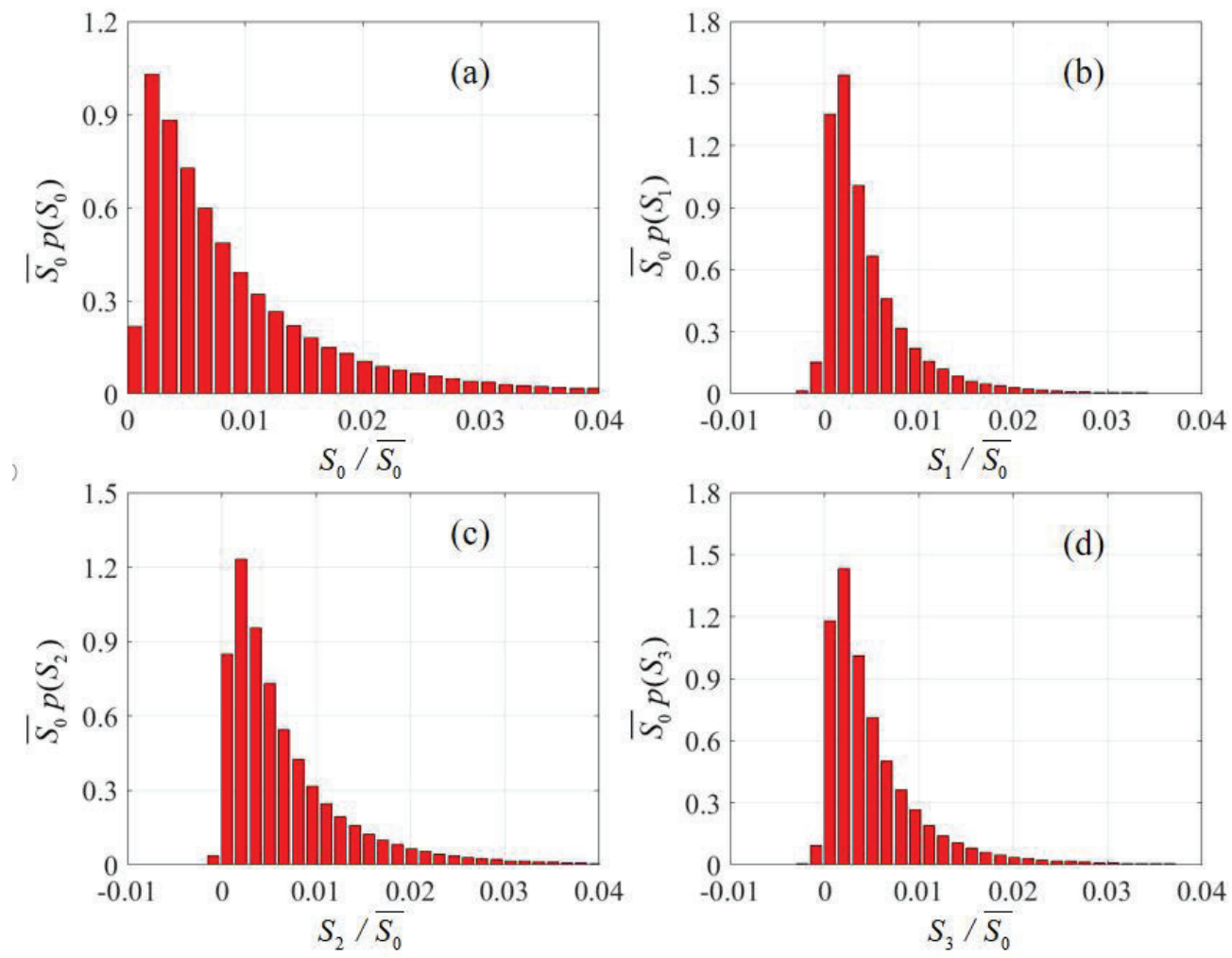

Figure 2. The histograms of the Stokes parameters for $S_{0}$ (a), $S_{1}$ (b), $S_{2}$ (c), and $S_{3}$ (d) 


\subsection{Ensemble-average Van Cittert-Zernike theorem for the Generalized Stokes parameters}

In Section 2, we introduced the concept of ensemble-average polarization coherence as a useful concept when dealing with non-ergodic statistical processes. A stationary polarization speckle pattern is such a process, for the time average of a stationary polarization speckle does not equal the average over an ensemble of possible polarization speckle patterns.

Since there is a difference between ensemble averages and time averages for this type of stochastic electric field, we must be careful to distinguish between time-averaged polarization coherence and ensemble-averaged polarization coherence. Accordingly, we shall use the ordinary symbols for polarization coherence quantities defined by time averages and identical symbols with overbars to represent ensemble-averaged quantities. Thus, we distinguish between two Generalized Stokes parameters: $S_{l}\left(\mathbf{r}_{1}, \mathbf{r}_{2}, \tau\right)$ and $\overline{S_{l}}\left(\mathbf{r}_{1}, \mathbf{r}_{2}, \tau\right)$ for $(l=0 \sim 3)$.

The wave equation governing the propagation of light is, of course, the same whether we are ultimately interested in time-average or ensemble-average properties of light. From this fact follows an important conclusion: the laws governing the propagation of coherence functions are identical for time-averaged and ensemble-averaged quantities. In other words, while the functional form of a mutual coherence may depend on whether the average is with respect to time or the ensemble, the mathematical relationship between two coherence functions of the same type is independent of which kind of averaging is used. ${ }^{[2]}$ This fact allows us to apply all our previously acquired knowledge of the propagation of ordinary polarization coherence functions to problems involving propagation of ensemble-average polarization coherence.

From an ensemble-averaging point of view, the Generalized Stokes parameters of the light reflected from or scattered by a rough-surfaced birefringent material, and observed very close to that surface, is essentially the same as the Generalized Stokes parameters of an incoherent source. Over an ensemble of ideally rough surfaces (i.e., surfaces with a very short correlation width of surface height fluctuations) there is little relationship between the phases of two polarization components of the light scattered from two closely spaced surface elements, at least until the spacing becomes close to a wavelength of the illuminating light. We state this fact mathematically by representing the ensembleaverage Generalized Stokes parameters of the light just leaving the surface by

$$
\overline{S_{l}}\left(\xi_{1}, \eta_{1} ; \xi_{2}, \eta_{2}\right)=\kappa \overline{S_{l}}\left(\xi_{1}, \eta_{1}\right) \delta\left(\xi_{1}-\xi_{2}, \eta_{1}-\eta_{2}\right),
$$

where $\kappa$ is a constant with dimensions meters squared, $\overline{S_{l}}$ is a distribution for the ensemble-average Generalized Stokes parameters, and $\delta$ is a two-dimensional delta function. A comparison with Eq, (5) shows that over an ensemble of possible reflected or scattered electric fields. the light leaving the rough-surfaced birefringent materials has ensemble properties that are identical with the time-averaged properties of completely spatially incoherent light. In effect, the light reflected at the surface behaves over the ensemble as spatially incoherent light, even though, by its time-averaged properties, the light is fully spatially coherent.

This fact allows us to make use of the Van Cittert-Zernike theorem to calculate the propagation of ensemble-averaged polarization coherence away from such a rough-surfaced birefringent material. In particular, for free-space propagation, we can write

$$
\left.\overline{S_{l}}\left(x_{1}, y_{1} ; x_{2}, y_{2}\right)=\frac{\kappa e^{-j \psi}}{(\bar{\lambda} z)^{2}} \iint_{-\infty}^{\infty} \overline{S_{l}}(\xi, \eta) \exp \left\{j \frac{2 \pi}{\bar{\lambda} z}\left[\left(x_{2}-x_{1}\right) \xi+\left(x_{2}-x_{1}\right) \eta\right)\right]\right\} d \xi d \eta,
$$

where $\psi=\pi\left[\left(x_{2}^{2}+y_{2}^{2}\right)-\left(x_{1}^{2}+y_{1}^{2}\right)\right] /(\bar{\lambda} z)$, and $\left(x_{m}, y_{m}\right)$ for $(m=1,2)$ are the coordinates in the observation plane with some distance $z$ from the rough surface and $\overline{S_{l}}$ is the distribution of the ensemble-averaged Stokes parameter of the light leaving the scattering spot. Thus, up to scaling constants, the ensemble-average Generalized Stokes parameters of the observed polarization speckle is given by a Fourier transform of the distributions of the average Stokes parameters leaving the rough surface. Thus, in free-space propagation, the ensemble-average mutual intensity in the observation plane can be found by means of a suitably scaled Fourier transform of the effective distributions of the Stokes parameters of the source. 

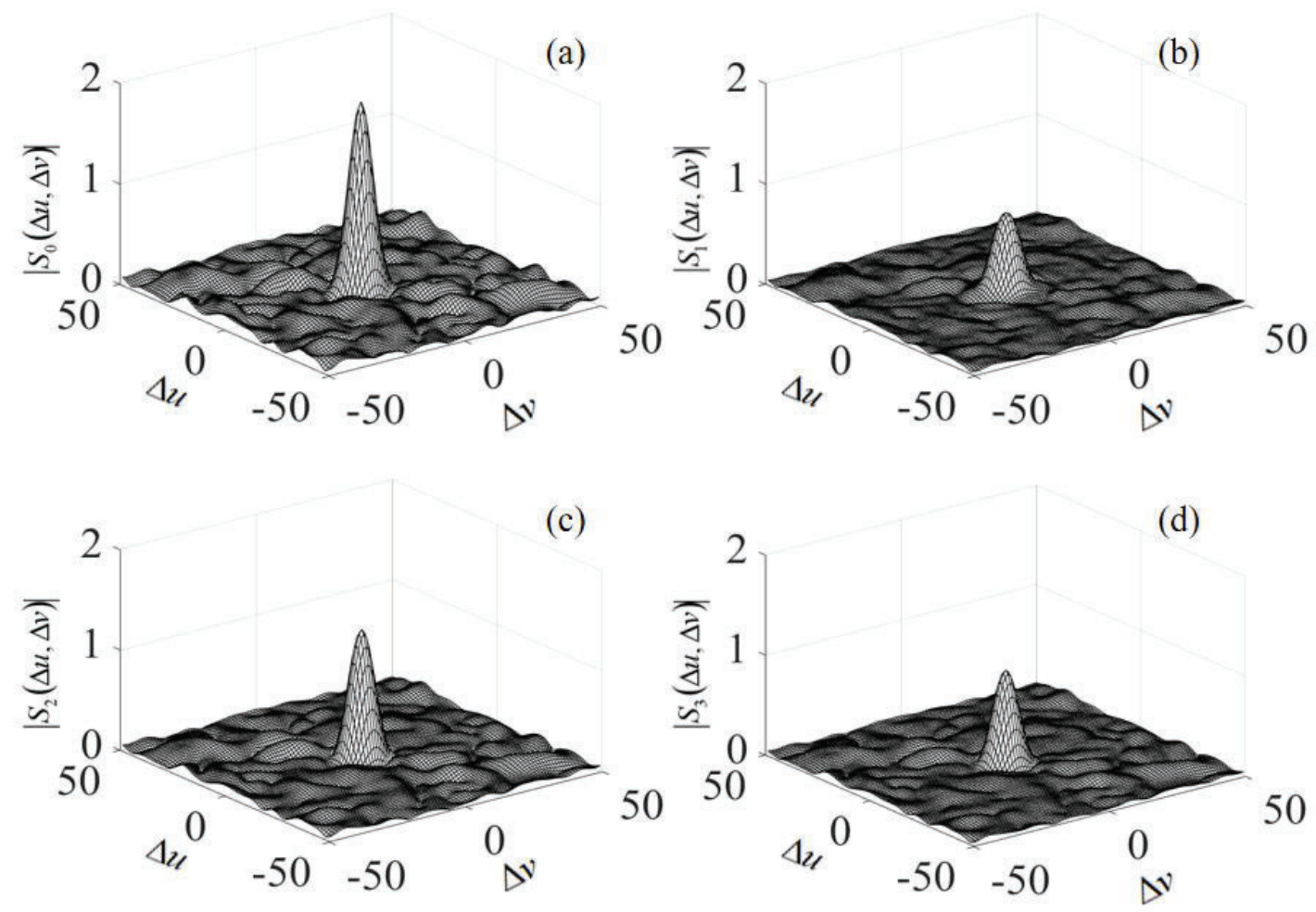

Figure 3. Experimental demonstration for the modulus of the ensemble-average Generalized Stokes parameters

From the reconstructed electric fields for polarization speckle at far field, we perform correlation calculation based on the definition of the Generalized Stokes parameters, where the ensemble-average in Eq. (7) has been replaced by spatial average. Figure 3 gives the modulus of the ensemble-average Generalized Stokes parameters.

\section{CONCLUSION}

Noting the existence of an important class of problems where time and ensemble averages yield different results, we propose a concept referred to as ensemble-average polarization and coherence for statistical optics and give the definition and physical indication of the ensemble-average degree of polarization for polarization speckle. Some statistics associated with polarization speckle including the 1st order statistical statistics of the Stokes parameters and the Generalized Stokes parameters are investigated theoretically and experimentally to demonstrate their very different statistical features.

\section{REFERENCES}

[1] J. W. Goodman, Statistical Optics 2nd edition, Wiley-Interscience, New York, (2015).

[2] L. Mandel and E. Wolf, Optical Coherence and Quantum Optics, Cambridge University Press, (1995).

[3] J. Dainty, Laser Speckle and Related Phenomena, Springer-Verlag, (1984).

[4] J. W. Goodman, Speckle phenomena in Optics: Theory and Applications $2^{\text {nd }}$ Edition, SPIE, (2017).

[5] A. F. Fercher and P. F. Steeger, "First-order statistics of Stokes parameters in speckle fields," Optica Acta 28, 443-448 (1981).

[6] P. F. Steeger and A. F. Fercher, "Experimental investigation of the first-order statistics of Stokes parameters in speckle fields," Optica Acta 29, 1395-1400 (1982).

[7] R. Barakat,"Statistics of the Stokes parameters," J. Opt. Soc. Am A 4(7), 1256-1263 (1987).

[8] R. Barakat,"The statistical properties of partially polarized light," Optica Acta 32(3), 295-312 (1985).

[9] D. Eliyahu,"Vector statistics of correlated Gaussian fields," Phys. Rev. E 47(4), 2881-2892 (1993). 
[10] D. Eliyahu, “Statistics of Styokes variables for correlated Gaussian field,” Phys. Rev. E 50(3), 2381-2384 (1994).

[11] R. Touzi and A. Lopes, "Statistics of the Stokes parameters and of the complex coherence parameters in one-look and multilook speckle fields," IEEE Trans. Geo. Rem. Sens. 34(2) 519-531 (1996).

[12] Y. Ohtsuka and K. Oka, "Contour mapping of the spatiotemporal state of polarization of light," App. Opt. 33(13), 2633-2636 (1994)

[13] W. Wang, S. G. Hanson, and M. Takeda, "Statistics of polarization speckle: Theory versus experiment," Proceedings of SPIE The International Society for Optical Engineering 7388(2009).

[14] M. Takeda, H.Ina, and S. Kobayashi, "Fourier-transform method of fringe-pattern analysis for computer-based topography and interferometry," J. Opt. Soc. Am. 72, 156-160 (1982). 